\title{
Commentary: An unusual case of crossing coronaries
}

\author{
Michael D. Taylor, MD, PhD
}

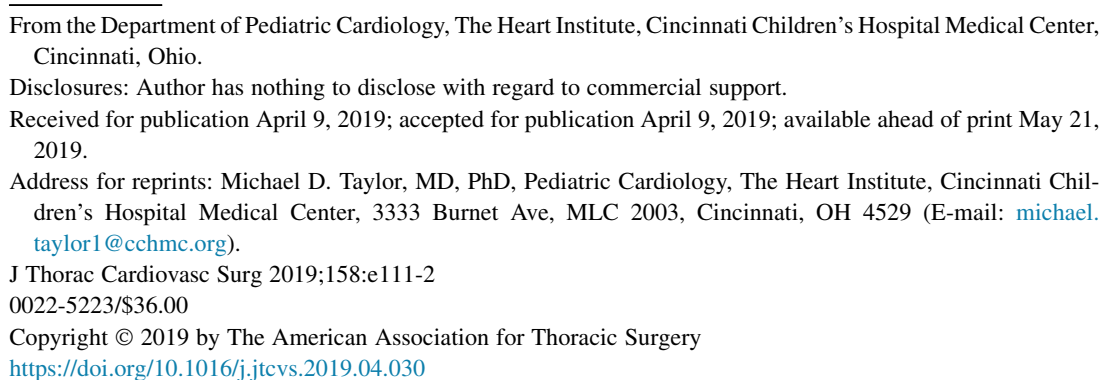

The coronary artery anatomy variations seen in transposition of the great arteries are important in planning and outcomes of the arterial switch operation (ASO). There are a variety of patterns, but one rule-understood to be universal-is that major epicardial coronaries do not cross to reach their myocardial distributions. The case report of Michalak and colleagues ${ }^{1}$ in this issue of the Journal describes a unique and previously unreported coronary pattern, with crossing coronary arteries in transposition of the great arteries after ASO as an infant. Interestingly, the anatomy was not discovered until follow-up coronary imaging when the patient was 20 years old. The case report highlights the seemingly infinite variation in coronary anatomy, provides clinical context to the current understanding of coronary development, and adds uncertainty to the debate regarding coronary surveillance for this patient group.

The variant described in the case report is historically unknown. The right and circumflex coronary arteries originated from right facing sinus, and the left anterior descending coronary artery originated from the left facing sinus. The coronaries were reimplanted in the rightward facing sinus of the neoaorta. Unknown to the surgeon at the time, the circumflex coronary reached its myocardial territory by traveling anterior to the main pulmonary artery and crossing the left anterior descending coronary artery.

This novel coronary pattern does not fit the dogma that coronary arteries are formed by channels growing toward the aortic sinus, because it would be difficult to hypothesize the channels crossing during development. It is more likely that crossing coronaries would only occur if the proximal coronaries grew outward from the sinuses. This is supported by newer histologic studies showing coronary artery stems originating from the aorta root in a 44-day embryo. ${ }^{2}$ Although understanding coronary artery embryology likely will not affect ASO outcomes, it may change how we understand diseases such as pulmonary atresia with intact septum or isolated coronary ostial atresia.

The most interesting clinical issue raised by the report regards the frequency and modality of ASO follow-up

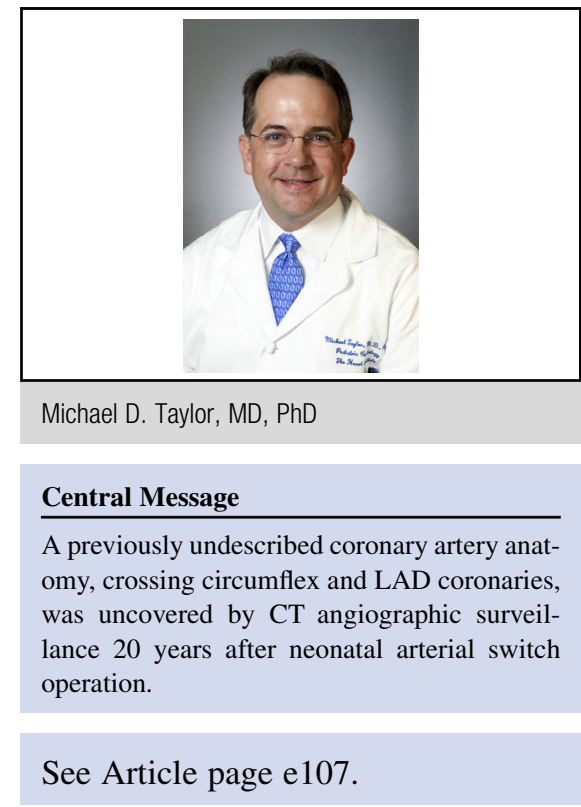

coronary imaging. The coronary arteries are at risk for obstruction, kinking, compression, and geometry changes with interval growth of the pulmonary arteries and neoaortic root. With the primary goal of preventing sudden death, recent guidelines recommend only that patients undergo coronary imaging once before adulthood. ${ }^{3}$ Conversely, in this report Michalak and colleagues ${ }^{1}$ advocate for computed tomographic angiography at prescribed intervals, but there is little evidence to support that this changes outcomes. Their group has found postsurgical changes in the coronary origins and geometry, luminal narrowing, and unknown patterns. It remains unclear, however, whether any of these findings carry functional significance. Most importantly, it is unknown whether they are substrates for adverse events in patients without symptoms. Because these findings can result in unnecessary cardiac catheterization, it is paramount that we understand the implications of these computed tomographic findings. The novel anatomy described is fascinating, but the clinical questions that the report asks is what the field should be doing for routine coronary surveillance in patients after ASO, and, perhaps more importantly, what clinical outcomes are associated with specific findings.

\section{References}

1. Michalak KW, Wernovsky G, Moll M, Anderson RH. The black swan: unique coronary arterial anatomy observed in a patient with transposition. J Thorac Cardiovasc Surg. 2019; 158:e107-9. 
2. Tomanek R, Angelini P. Embryology of coronary arteries and anatomy/pathophysiology of coronary anomalies. A comprehensive update. Int J Cardiol. 2019;281:28-34.

3. Cohen MS, Eidem BW, Cetta F, Fogel MA, Frommelt PC, Ganame J, et al. Multimodality imaging guidelines of patients with transposition of the great arteries: a report from the American Society of Echocardiography developed in collaboration with the Society for Cardiovascular Magnetic Resonance and the Society of Cardiovascular Computed Tomography. J Am Soc Echocardiogr. 2016;29:571-621. 